UCRL-JC-127113

PREPRINT

\title{
Adapting the Decomposable Mandrel Technique To Build Specialty ICF Targets
}

\author{
E.M. Fearon \\ S.A. Letts \\ L.M. Allison \\ R.C. Cook
}

This paper was prepared for submittal to the Eleventh Target Fabrication Specialists' Meeting

Orcas Island, Washington

September 8-12, 1996

March 26, 1997

This is a preprint of a paper intended for publication in a journal or proceedings. Since changes may be made before publication, this preprint is made available with the understanding that it will not be cited or reproduced without the permission of the author. 


\section{DISCLAIMER}

This document was prepared as an account of work sponsored by an agency of the United States Government. Neither the United States Government nor the University of California nor any of their employees, makes any warranty, express or implied, or assumes any legal liability or responsibility for the accuracy, completeness, or usefulness of any information, apparatus, product, or process

disclosed, or represents that its use would not infringe privately owned rights. Reference herein to any specific commercial product, process, or service by trade name, trademark, manufacturer, or otherwise, does not necessarily constitute or imply its endorsement, recommendation, or favoring by the United States Government or the University of California. The views and opinions of authors expressed herein do not necessarily state or reflect those of the United States Government or the University of California, and shall not be used for advertising or product endorsement purposes. 


\title{
ADAPTING THE DECOMPOSABLE MANDREL TECHNIQUE TO BUILD SPECIALTY ICF TARGETS
}

\author{
Evelyn M. Fearon, Stephan A. Letts, \\ Leslie M. Allison, and Robert C. Cook \\ Lawrence Livermore National Laboratory, \\ Livermore CA 94551
}

(510)423-1817

\section{ABSTRACT}

In this paper we describe our efforts to produce ICF target capsules with either controlled inner surface roughness or thin metallic diagnostic layers by adapting the decomposable mandrel technique previously developed at LLNL. To modify the capsule's inner surface we laser ablated a pattern on a poly ( $\alpha$-methylstyrene) (PAMS) shell, overcoated it with plasma polymer and then thermally decomposed the inner mandrel to leave the plasma polymer shell with the imprint of the laser ablated mandrel pattern. In this fashion we have been able to produce shells with controlled inner surface bumps. However, these bumps are correlated with outer surface pits. To place a thin metallic diagnostic layer on the inner capsule surface we applied a $50 \AA$ titanium sputter coating to a smooth PAMS shell, overcoated with plasma polymer, and then thermally decomposed the mandrel to leave a plasma polymer shell with the titanium layer on the inner surface. Surface analysis showed that this process resulted in shells with a relatively long wavelength roughness, possibly due to the action of the metallic layer as a permeation barrier.

\section{INTRODUCTION}

We have previously reported on a new method of producing high quality capsules for ICF experiments that utilizes a decomposable mandrel. .,2,3 $^{1,3}$ The central row of Figure 1 shows the basic process. A shell of poly( $\alpha$-methyl styrene) (PAMS) produced by microencapsulation is overcoated with plasma polymer $(\mathrm{CH}) .{ }^{4}$ This composite shell is then heat treated at $300{ }^{\circ} \mathrm{C}$ to decompose the PAMS to gaseous monomer which diffuses through the thermally more stable plasma polymer shell to leave a shell which replicates the initial mandrel.

Our interest in the work reported here was to determine if this technique could be used to produce specialty capsules, specifically ones with a controlled inner surface

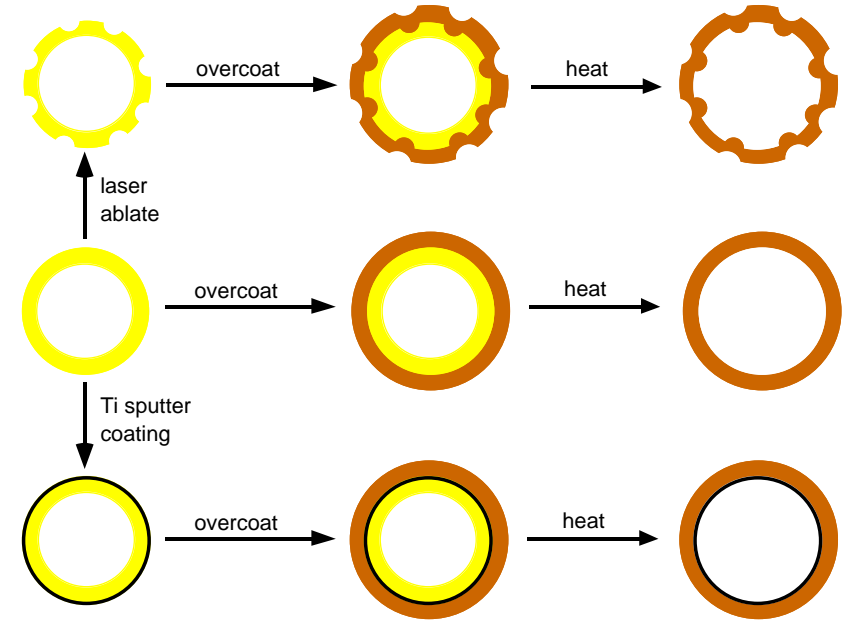

Figure 1. The center row illustrates the basic PAMS mandrel technique of overcoating a PAMS shell with plasma polymer and then thermally treating it to depolymerize the PAMS, leaving a hollow shell. The first and last rows depict the variations to produce the special target shells with inner surface roughness and a metal layer on the inside surface.

roughness or a thin metallic diagnostic layer on or near the inner capsule wall. The ability to control the surface finish of the capsule interior would allow us to test the effects of inner surface roughness on capsule performance, experimentally verifying our understanding of this aspect of the implosion hydrodynamics, similar to what has been done with outer surface roughness experiments. ${ }^{5,6}$ Thin $(50 \AA)$ metallic diagnostic layers on or controllably near the capsule inner surface (in contradistinction to a low doping level throughout the inner $3 \mu \mathrm{m}$ of wall) offers the possibility of improved interpretation of the time dependent mix induced spectroscopic signal. ${ }^{7}$

The proposed routes to these capsules are outlined in the top and bottom rows of Figure 1. For controlled inner surface roughness (top row, Figure 1) we proposed to laser ablate a pattern on the PAMS mandrel before overcoating 
and thermal decomposition. In this way the pattern of pits on the mandrel would appear as bumps on the inside of the resulting plasma polymer shell (as well as pits on the shell exterior). To produce a thin metallic diagnostic layer (bottom row Figure 1) we proposed to sputter coat a thin metal layer on the PAMS mandrel before plasma polymer overcoating and thermal treatment so that it would be transferred to the inside of the resulting plasma polymer shell. The key issue here is clearly the ability of the PAMS decomposition products to diffuse through such a metallic layer.

In what follows we will discuss each of these efforts and the results obtained. In neither case were we completely sucessful in producing a useful target, but the studies were valuable in exploring the capabilities and limitations of the PAMS technique.

\section{TEXTURED INNER SURFACE}

\section{A. Experimental}

Preliminary laser ablation and plasma polymer overcoat experiments were performed on flat substrates. Silicon wafers were first coated with several microns of plasma polymer, and then a pattern of 1 and $2 \mu \mathrm{m}$ deep pits, $100 \mu \mathrm{m}$ in diameter, were laser ablated on the surface. Individual pits were mapped with an Atomic Force Microscope (AFM), and then 70 additional $\mu \mathrm{ms}$ of plasma polymer were applied. The pits were then remapped and found to have the same depth and width as previously measured, confirming that the coating was applied with uniform thickness to the surface. It is worth noting that the uniform coating model predicts pit filling from the edge, but only after adding a coating thickness on the order of the radius of curvature of the pit, which in this case is the order of $1000 \mu \mathrm{m}$.

The spherical substrates used were microencapsulated PAMS shells. A number of nearly identical pits were laser ablated in a regular pattern on one hemisphere of the shell surface. ${ }^{8}$ The pits were characterized by using the AFMbased profilometer (Sphere Mapper) ${ }^{9}$ as a "band mapper". By taking 25 sequential equatorial traces each separated from the next by $5 \mu \mathrm{m}$, a $125 \mu \mathrm{m}$ wide surface "ribbon" map was produced around the sphere equator which included a number of the ablated pits. Two $35 \mu \mathrm{m}$ layers of plasma polymer were then added to the shells, with band mapping after each. Some of the overcoated shells with $35 \mu \mathrm{m}$ of coating were pyrolyzed to remove the PAMS mandrel and then ribbon mapped.

\section{B. Results and Discussion}

An example of part of a ribbon map from one of the shells after pyrolysis of the underlying PAMS is shown in Figure 2. The pit widths are close to the $125 \mu \mathrm{m}$ band map width, thus, there are several incompletely imaged pits. Unlike the ablated flats, the difficulty of exactly reorienting the sphere on the Sphere Mapper prevented us from obtaining dimensions of the same pit before and after overcoating. Thus we can only look for qualitative changes in the pits after each step in the experiment.

Table I lists diameter and depth measurements for pits at each stage of the experiment where at least $70 \%$ of the pit area was imaged. As explained above, measurements in any

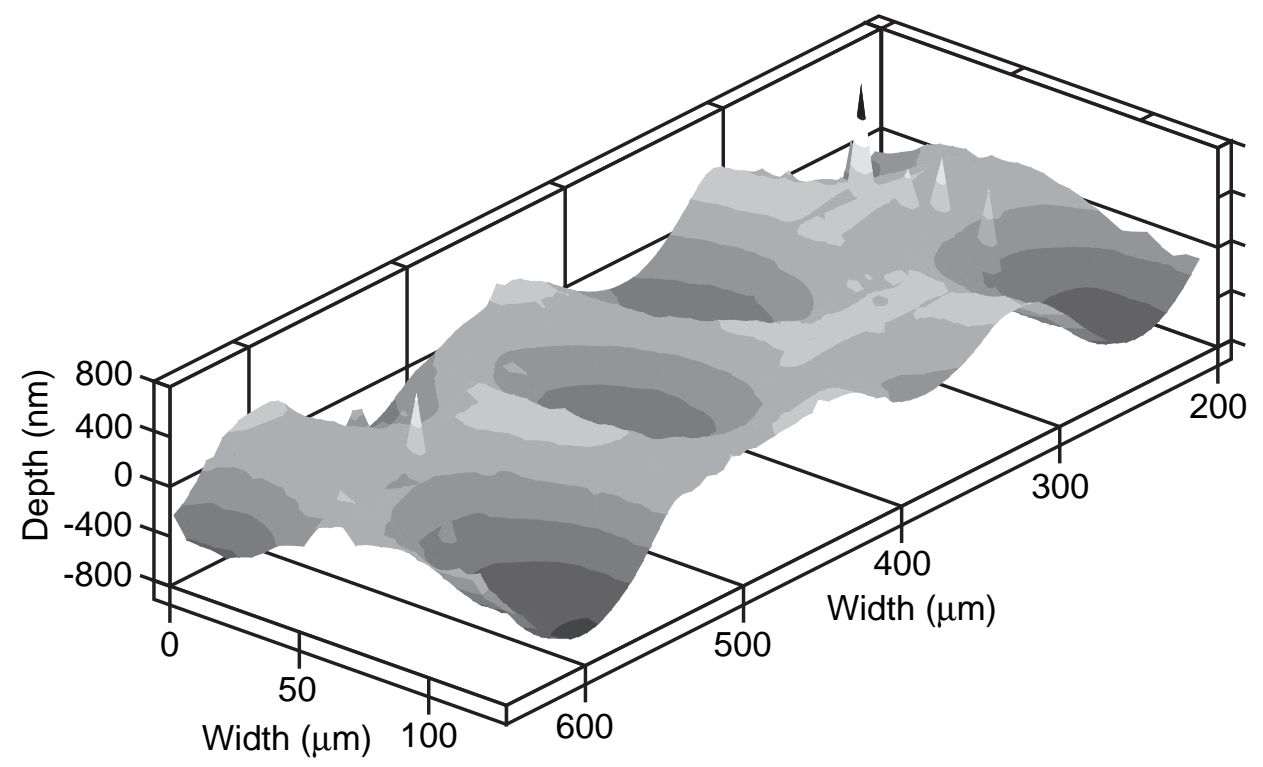

Figure 2. A band map from a pyrolyzed, textured shell. The pits are actually circular; the dissimilar axis scales make them appear oval. The map also shows some examples of surface debris spikes. 
Table I A selection (from a variety of shells) of representative diameter and depth measurements (in $\mu \mathrm{m}$ ) of pits as ablated, after 35 and $70 \mu \mathrm{m}$ of plasma polymer overcoat, and after shells with $35 \mu \mathrm{m}$ of overcoat were pyrolyzed to remove the PAMS mandrel. Note that the measurements in any line do not represent the same pit or even the same shell.

\begin{tabular}{lllllllll}
\multicolumn{2}{l}{ as ablated } & \multicolumn{2}{c}{$+35 \mu \mathrm{m} \mathrm{CH}$} & \multicolumn{2}{c}{$+70 \mu \mathrm{m} \mathrm{CH}$} & \multicolumn{2}{l}{$+35 \mu \mathrm{m} /$ pyrolyzed } \\
$\underline{\text { diam }}$ & $\underline{\text { depth }}$ & $\underline{\text { diam }}$ & $\underline{\text { depth }}$ & $\underline{\text { diam }}$ & $\underline{\text { depth }}$ & $\underline{\text { diam }}$ & $\underline{\text { depth }}$ \\
120 & 0.61 & 124 & .074 & 135 & .0 .71 & 125 & 0.74 \\
105 & 0.63 & 140 & 0.84 & 132 & 0.79 & 118 & 0.62 \\
132 & 0.66 & 124 & 0.79 & 135 & 0.68 & 128 & 0.64 \\
& & & & 140 & 0.74 & & \\
119 & 0.63 & 129 & 0.79 & 136 & 0.73 & 124 & 0.67
\end{tabular}

line do not represent the same pit. Average values show that there was clearly no significant modification of the pit depth after coating or mandrel pyrolysis. The apparent increase in diameter for the shells overcoated with $70 \mu \mathrm{m}$ of $\mathrm{CH}$ is probably due both to poor statistics and to the uncertainty in identifying the pit boundary in the overcoated shells. Most importantly, the coated shells that were heat treated show no significant change in pit dimensions.

AFM characterization of the inside surface of the plasma polymer shell was not attempted. However, note that since the measured outside surface of the pyrolyzed shells shows pits of approximately the same dimensions as the originally ablated ones, and since the plasma polymer has been shown to be uniformly applied, the pits on the outside must mirror bumps on the inside.

We have shown that laser ablated pits on the surface of a microencapsulated PAMS mandrel do not change dimension when layers of plasma polymer up to $70 \mu \mathrm{m}$ thick are added. They also do not change when the overcoated shell is pyrolyzed to remove the underlying PAMS and produce a plasma polymer shell. This method, then, can produce targets with inner bumps and perfectly correlated outer depressions. To produce a smooth outer surface on these targets, a coating technique would have to be developed to fill in the surface pits. Some possibilities might involve a solvent casting technique or the melting of a thermoplastic overcoating layer where in each case the surface tension would drive the liquid layer to smooth over the pits.

\section{TITANIUM DIAGNOSTIC LAYER}

\section{A. Experimental}

A titanium (Ti) layer was chosen for our experiments because Ti has been recently incorporated into Nova polystyrene mandrels for use as a spectroscopic marker. ${ }^{10} \mathrm{~A}$ Ti layer thickness of $50 \AA$ was used in the work described here because that is the equivalent pure metal thickness to 0.1 atom $\%$ Ti dispersed in a $3 \mu \mathrm{m}$ thick polystyrene mandrel used in recent Nova targets.

The titanium was applied to microencapsulated PAMS shells in a magnetron sputtering deposition system. The system was run at $10^{-7}$ torr chamber pressure, $0.5 \mathrm{amp}$ current for 1.1 to 2 minutes for $50 \AA$ thickness. A rate monitor was used in the coater to determine coating time to reach $50 \AA$. Actual metal thickness was determined after coating by measuring Ti content (mass) in the shell by $\mathrm{x}$-ray microfluorescence (XRMF). We experienced a $40 \%$ variation in actual Ti mass for a rate monitor reading of $50 \AA$ in this experiment. After adding a plasma polymer coating over the Ti layer, the composite shell was heat treated to remove the underlying PAMS mandrel. The prepyrolyzed composite mandrels and postpyrolyzed shells were fully characterized by optical and scanning electron microscope (SEM), Sphere Mapper traces, and x-ray microfluorescence for Ti content.

\section{B. Results and Discussion}

By mass and dimensional measurements before and after heat treatment we determined that the underlying PAMS mandrel was in fact being removed. Thick walled $(38 \mu \mathrm{m})$ product shells looked uniform and spherical optically. However, the thin walled shells showed some non-uniformity when examined optically. The Sphere Mapper revealed shell deformation. Figure 3 shows representative equatorial traces of shells from the same $\mathrm{Ti}$ and plasma polymer coating batch before and after pyrolysis. The shells in the first two sets of traces of the figure have nominally $50 \AA$ Ti inside a $6 \mu \mathrm{m}$ thick plasma polymer shell. The third set of traces, shown for comparison, is from a pyrolyzed plain plasma polymer shell with a $4 \mu \mathrm{m}$ thick wall. The figure shows that the deformation does not occur in either the Ti coater or the plasma polymer coater prior to heat treatment, but rather is directly associated with the pyrolysis of a Ti coated PAMS mandrel.

Figure 3 is representative of all the Ti layered shells studied, regardless of plasma polymer overcoat thickness. 


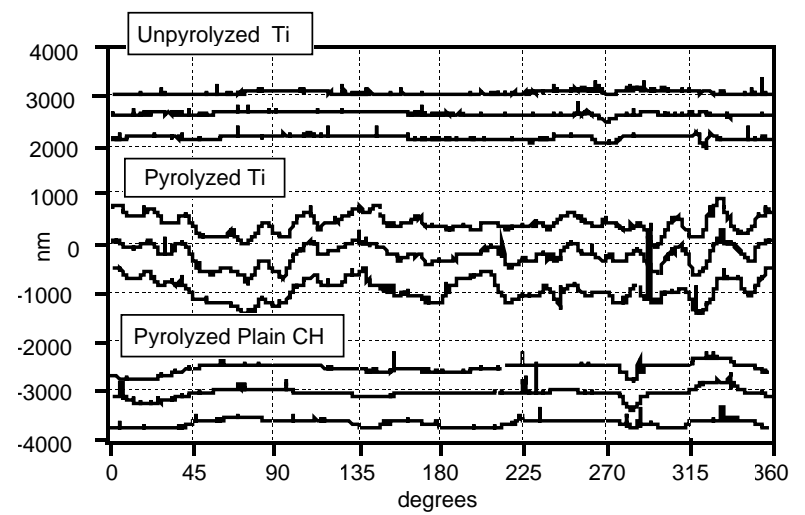

Figure 3. Sphere Mapper equatorial traces of Ti coated shells before and after pyrolysis show that the shell deforms upon heat treatment. The third set of traces shows a pyrolyzed shell from a plain PAMS mandrel, showing there is no deformation from the heat treatment of shells without the Ti layer.

Note that the ordinate unit of degrees around the circumference of the shell represents $4 \mu \mathrm{m}$ per degree for these roughly $0.5 \mathrm{~mm}$ diameter shells. The amplitude of the long wavelength deformations present on the baked Ti coated shell is only 0.5 to $1 \mu \mathrm{m}$, however, making optical measurement techniques inadequate to detect their presence in thick walled shells.

It is clear that the deformations are linked in some fashion to the pyrolysis process, possibly due to the Ti layer acting as a permeation barrier to the PAMS decomposition products. It is not clear how this results in long wavelength deformations, however.

Different methods were tried to reduce the deformation effect. We first studied the effect of a change in the furnace temperature ramp rate. The standard ramp rate for decomposing the PAMS shells is $1 \% \mathrm{~min}$., which worked well for the non-Ti coated runs. Slower rates of $0.1 \% \mathrm{~min}$. and $0.03 \% \mathrm{~min}$. were tried for the Ti coated shells. Unfortunately, the equatorial traces of these shells were similar to those shown in Figure 3.

We then tried to change the nature of the Ti layer so that it might be more permeable. Varying the chamber coating pressure from $10^{-7}$ torr (the standard chamber pressure before beginning the sputtering run) to $10^{-5}$ torr was tried. At the higher pressure, it is thought that the sputtered metal combines with residual oxygen in the chamber or with water desorbing from the chamber walls and the coating is produced as the metal oxide. The hope was that the metal oxide would be more porous than the pure metal. However, surface analysis of shells from these experiments showed there was no benefit from higher chamber pressure for the deformation problem.

We also tried varying the composition of the surface upon which the Ti was applied. To accomplish this a $0.5 \mu \mathrm{m}$ layer of plasma polymer was applied to the PAMS shell before coating with Ti. The final composite shell had $33 \mu \mathrm{m}$ of plasma polymer overcoating and a Ti thickness of $15 \AA$, as measured by XRMF. This was an anomalously low Ti thickness. It is not known if it was due to low sputtering flux or to the different surface material. We found, however, that there was still some surface deformation in the pyrolyzed shells, but it was not as severe as with previous experiments.

In order to examine more carefully whether the $\mathrm{Ti}$ coating was acting as a permeation barrier to the PAMS decomposition products, an experiment was performed to directly measure the prepyrolyzed permeation properties of the Ti coated shells. Using PAMS shells from the same batch, some were left bare and $50 \AA$ of Ti (verified by XRMF) was applied to others. Each set was placed in an argon (Ar) pressure vessel for 16 hours at 100 psi in order to fill the shells with Ar. We then measured by XRMF the decay of the Ar x-ray counts versus time as the Ar leaked out of individual shells, with and without the Ti layer. Figure 4 is a plot of the Ar signal, which is proportional to the pressure or Ar gas inside the shell, as a function of time. The circle symbol denotes the bare shells; the triangle symbols are the shell plus $50 \AA$ of titanium.

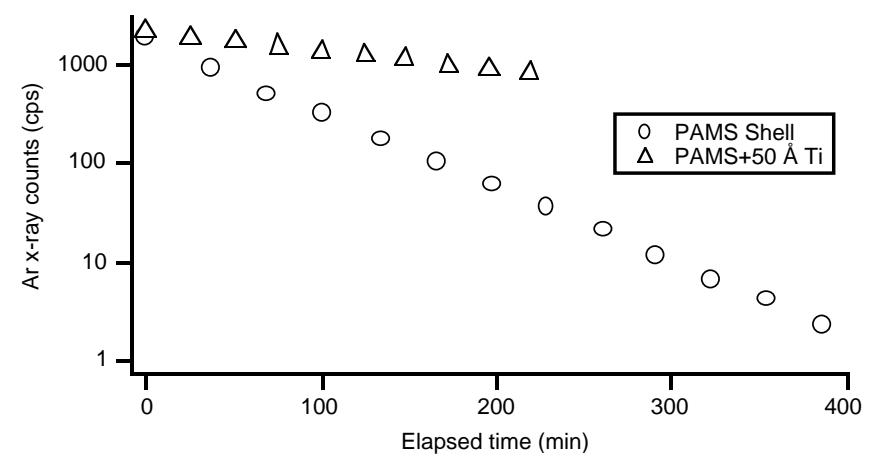

Figure 4. Argon hold time of titanium coated PAMS shells compared with uncoated PAMS shells.

The straight line decay in Figure 4 on a semi-log plot indicates that the depressurization is following a first-order rate process. The pressure of gas remaining in the shell can thus be expressed as

$$
P(t)=P_{0} \exp \left(-\frac{0.693 t}{\tau_{1 / 2}}\right)
$$

where $P(t)$ is the pressure in the shell at time $t, P_{0}$ is the initial pressure in the shell, and $\tau_{1 / 2}$ is the pressure half-life, the length of time it takes for the pressure in the shell to reach one-half the initial pressure. The slope of the lines in Figure 4 is easily related to $\tau_{1 / 2}$. 
Clearly, the $50 \AA$ A thick Ti coating did act as a permeation barrier, compared with the bare shells. The half life increased from about 35 minutes for the bare shells to about 150 minutes for the Ti coated shells.

Although there is a permeation effect of the Ti layer, it is not apparent that the factor of five decrease in permeation due to the layer is the cause of the shell deformation, particularly in view of the pyrolysis experiments performed at very slow temperature ramp rates which had no effect on the deformation. Further, as noted above, it is not clear how a decreased permeation capability leads to low amplitude long wave length deformations such as those shown in Figure 3. Let us conclude this section with two different conjectures as to the cause of the deformation. One possibility is that perhaps the Ti layer is not continuous, and thus certain regions of the shell surface are more permeable than others. This would result in different fluxes of decomposition products through different portions of the shell which might lead to shell deformation. A second possibility is as follows. It is known that the pyrolysis step for standard shells leads to a roughly $5 \%$ uniform shrinkage of the shell. ${ }^{11}$ Might it be that the difference in shrinkage between the plasma polymer layer and the Ti layer leads to the deformation? We have no evidence for either of these conjectures, but should there be a renewed interest in thin metal diagnostic layers for ICF capsule experiments more thorough experiments to test these and other ideas will be necessary.

\section{ACKNOWLEDGMENTS}

The authors thank Ed Lindsey, Craig Moore, Andrea Denton-Paul, Rand McEachern, Bob Turner, and Charlotte King for providing analytical support;

Ricke Behymer for many titanium coating runs; Bill Wilcox for performing the laser photoablation. We also thank Fred Elsner and Don Czechowicz of General Atomics for providing microencapsulated shells. This work was performed under the auspices of the U.S. Department of Energy by Lawrence Livermore National Laboratory under Contract W-7405-ENG-48.

\section{REFERENCES}

1. S. A. Letts, E. M. Fearon, S. R. Buckley, M. D. Saculla, L. M. Allison, and R. Cook, "Preparation of Hollow Shell ICF Targets using a Depolymerizing Mandrel," in Hollow and Solid Spheres and Microspheres - Science and Technology Associated with Their Fabrication and Application, edited by D. L. Wilcox, et. al. (Materials Research Society, Pittsburgh, PA, 1995), pp 125-130.

2. S. A. Letts, E. M. Fearon, S. R. Buckley, M. D. Saculla, L. M. Allison, and R. Cook, "Fabrication of Polymer Shells Using a Decomposable Mandrel," Fusion Technol. 28, 1797 (1995).
3. S. A. Letts, E. M. Fearon, L. M. Allison, and R. Cook, "Fabrication of Special Inertial Confinement Fusion Targets Using a Depolymerizable Mandrel Technique," J. Vac. Sci. Technol. A 14, 1015 (1996).

4. S. A. Letts, D. W. Myers, and L. A. Witt, "Ultrasmooth Plasma Polymerized Coatings for Laser Fusion Targets", J. Vac. Sci. Technol., 19, 739 (1981)

5. O. L. Landen, et. al., "Indirectly Driven, High Growth Rayleigh-Taylor Implosions on Nova," J. Quant. Spectrosc. Radiat. Transfer 54, 245 (1995).

6. O. L. Landen, et al., "High-Growth-Factor Implosions (HEP4)," ICF Quarterly Report 5, 271 (1995).

7. C. J. Keane, et al.," X-ray Spectroscopic Diagnostics of Mix in High Growth Factor Spherical Implosions," $J$. Quant. Spectrosc. Radiat. Transfer 54, 207 (1995).

8. R. J. Wallace, R. L. McEachern, W. W. Wilcox, "Laser Ablation Machining of ICF Capsules", LLNL ICF Quarterly Report, UCRL-LR-105821-4-3, 4(3) 79 (1994)

9. R. L. McEachern, C. E. Moore, and R. J. Wallace, "The Design, Performance, and Application of an Atomic Force Microscope-Based Profilometer", J. Vac. Sci Technol., A13, 983 (1995)

10. same as ref 7 (Keane et al)

11. same as ref 2 (Letts et al) 